\title{
INFRARED TRANSMISSION SPECTRA FOR EXTRASOLAR GIANT PLANETS
}

\author{
Giovanna Tinetti, ${ }^{1}$ MaO-Chang Liang, ${ }^{2,3}$ Alfred Vidal-Madjar, ${ }^{1}$ David Ehrenreich, ${ }^{1}$ \\ Alain Lecavelier des Etangs, ${ }^{1}$ And Yuk L. Yung \\ Received 2006 August 9; accepted 2006 November 8; published 2006 December 14
}

\begin{abstract}
Among the hot Jupiters known to date that transit their parent stars, the two best candidates to be observed with transmission spectroscopy in the mid-infrared (MIR) are HD 189733b and HD 209458b, due to their combined characteristics of planetary density, orbital parameters, and parent star distance and brightness. Here we simulate transmission spectra of these two planets during their primary transit in the MIR, and we present sensitivity studies of the spectra to the changes of atmospheric thermal properties, molecular abundances, and $\mathrm{C} / \mathrm{O}$ ratios. Our model predicts that the dominant species absorbing in the MIR on hot Jupiters are water vapor and carbon monoxide, and their relative abundances are determined by the $\mathrm{C} / \mathrm{O}$ ratio. Since the temperature profile plays a secondary role in the transmission spectra of hot Jupiters compared to molecular abundances, future primary transit observations in the MIR of those objects might offer insight on extrasolar giant planet atmospheric chemistry. We find here that the absorption features caused by water vapor and carbon monoxide in a cloud-free atmosphere are deep enough to be observable by the present and future generation of space-based observatories, such as Spitzer Space Telescope and James Webb Space Telescope. We discuss our results in light of the capabilities of these telescopes.
\end{abstract}

Subject headings: atmospheric effects — occultations — radiative transfer — techniques: spectroscopic

Online material: color figure

\section{INTRODUCTION}

Extrasolar giant planets (EGPs) are now being discovered at an accelerating pace (Schneider 2006; Butler et al. 2006). In particular, an increasing interest has been focused on hot Jupiters that transit their parent stars, since they represent a valuable tool to determine key physical and atmospheric parameters of the EGPs (Seager \& Sasselov 2000). The most studied transiting extrasolar planet, HD 209458b, orbits a main-sequence G-type star at $0.046 \mathrm{AU}$ (period 3.52 days). It is the first one for which repeated transits across the stellar disk were observed ( $1.6 \%$ absorption; Henry et al. 2000; Charbonneau et al. 2000). Along with radial velocity measurements (Mazeh et al. 2000), it was possible to determine mass and radius $\left(M_{p} \sim 0.69 M_{\mathrm{J}}, R_{p} \sim 1.4 R_{\mathrm{J}}\right)$, confirming that the planet is a gas giant with one of the lowest densities discovered so far. Owing to this property, its very extended atmosphere is one of the best candidates to be probed with transit techniques. In particular, the upper atmosphere extends beyond the Roche lobe, showing a population of escaping atoms (Vidal-Madjar et al. 2003, 2004). The numerous follow-up observations of HD 209458b also include the detection and upper limits of absorption features in the deeper atmosphere (Charbonneau et al. 2002; Richardson et al. 2003a, 2003b, 2006; Deming et al. 2005a). Most recently, Deming et al. (2005b) detected the thermal emission of this planet with Spitzer during a secondary transit in the $24 \mu \mathrm{m}$ band, and Richardson et al. (2006) detected the first primary transit in the same band. To explain these observations several models were proposed, including atmospheric photochemistry, thermal properties, three-dimensional (3D) circulation simulations, cloud and condensate height, and escaping processes (e.g. Fortney et al. 2003; Burrows et al. 2003; Liang et al. 2003; Lecavelier et al. 2004; Yelle 2004; Tian et al. 2005; Iro et al. 2005; Seager et al. 2005).

\footnotetext{
${ }^{1}$ Institut d'Astrophysique de Paris, CNRS, Université Pierre et Marie Curie, 75014 Paris, France; tinetti@iap.fr, alfred@iap.fr, ehrenreich@iap.fr, lecaveli@iap.fr.

${ }^{2}$ California Institute of Technology, Division of Geological and Planetary Sciences, Pasadena, CA 91125; mcl@gps.caltech.edu, yly@gps.caltech.edu.

${ }^{3}$ Research Center for Environmental Changes, Academia Sinica, Taipei 115, Taiwan.
}

Additional observations are required, however, to constrain the past, present, and future modeling effort. The planet HD 189733b, recently discovered by Bouchy et al. (2005) with mass $M_{p} \sim$ $1.15 M_{\mathrm{J}}$ and $R_{p} \sim 1.26 R_{\mathrm{J}}$, orbits an early main-sequence $\mathrm{K}$ star at $0.0313 \mathrm{AU}$. It is an exoplanet transiting the brightest and closest star discovered so far.

Here we focus our interest on both planets HD 209458b and HD 189733b, and we model the spectral absorption features in the mid-infrared (MIR) due to the most abundant atmospheric molecules during their primary transit, i.e., when the planet passes in front of the parent star.

In a solar system like ours, a significant amount of water vapor $\left(\mathrm{H}_{2} \mathrm{O}\right)$ can exist only in planetary atmospheres at orbital distances less than $1 \mathrm{AU}$. The requirement is certainly met for the known transiting EGPs. Carbon monoxide $(\mathrm{CO})$ and methane $\left(\mathrm{CH}_{4}\right)$, and other photochemical products, such as carbon dioxide $\left(\mathrm{CO}_{2}\right)$ and acetylene $\left(\mathrm{C}_{2} \mathrm{H}_{2}\right)$, are plausibly present in the atmospheres of EGPs and possibly abundant to be detected. These species have strong absorption bands in the MIR, and more importantly, in spectral regions compatible with present and future space-based observations such as Spitzer or the James Webb Space Telescope (JWST; Gardner et al. 2006). Given $\mathrm{O}$ and $\mathrm{C}, \mathrm{H}_{2} \mathrm{O}$ and $\mathrm{CO}$ will be controlled mainly by the relative abundances of these two species:

1. If the $\mathrm{C} / \mathrm{O}$ ratio is close to solar, $\mathrm{H}_{2} \mathrm{O}, \mathrm{CO}$, and $\mathrm{CH}_{4}$ abundances are determined by the thermodynamic equilibrium chemistry in the deep atmosphere (Liang et al. 2003, 2004).

2. If the $\mathrm{C} / \mathrm{O}$ ratio is above solar, according to the scenario proposed by Kuchner \& Seager (2006) planets should show a significant paucity of water vapor in their atmospheres, and carbon-rich species should by contrast be enhanced. $\mathrm{CO}$ is expected to be the dominant carbon-bearing molecule at high temperatures and $\mathrm{CH}_{4}$ at low temperatures.

3 . If the $\mathrm{C} / \mathrm{O}$ ratio is below solar, the atmosphere is depopulated of carbon-bearing molecules, and water-vapor is the dominant species between $\sim 10^{-10}$ and 1.5 bars. 

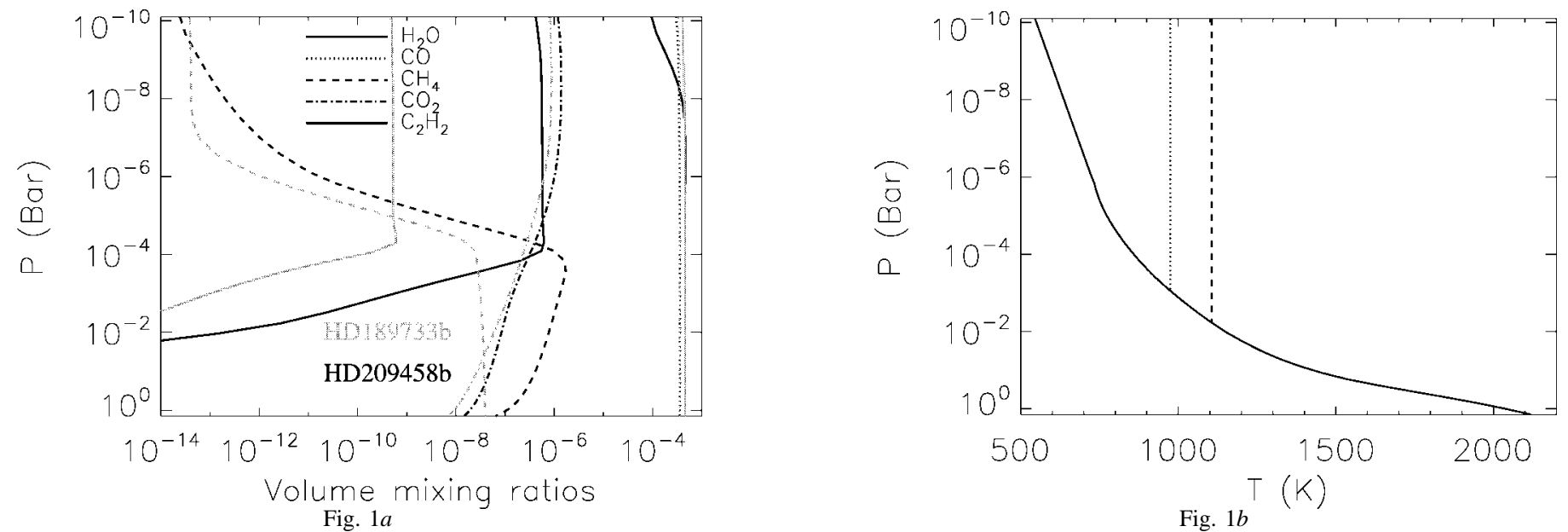

FIG. 1.-(a) Profiles of $\mathrm{H}_{2} \mathrm{O}$ (solid line), $\mathrm{CO}$ (dotted line), $\mathrm{CO}_{2}$ (dot-dashed line), $\mathrm{CH}_{4}$ (dashed line), and $\mathrm{C}_{2} \mathrm{H}_{2}$ (triple-dot-dashed line) for planets $\mathrm{HD}$ 209458b (black lines) and HD 189733b calculated with the photochemistry model described in Liang et al. (2003, 2004). (b)Temperature-pressure profiles used for our simulations. Solid line: Standard profile. Dotted line: Hot profile. Dashed line: Very hot profile. [See the electronic edition of the Journal for a color version of this figure.]

\section{DESCRIPTION OF THE MODEL}

We have built a model of the planetary atmosphere and calculated the expected absorption of the stellar light when filtered through the planetary atmospheric layers. This has already been discussed in the literature. In particular, for our simulations, we have used the geometry and the equations described in Brown (2001, their Fig. 1, configuration 2) and Ehrenreich et al. (2006, § 2.1; their Fig. 1). Our cloud and hazefree atmospheres were divided in 40 layers spanning from $\sim 10^{-10}$ to 1 bars.

Photochemical models are used to determine the molecular abundances of 33 species above $\sim 1$ bar altitude level. We start with four parent molecules $\mathrm{H}_{2}, \mathrm{CO}, \mathrm{H}_{2} \mathrm{O}$, and $\mathrm{CH}_{4}$. Their relative abundances are determined by thermochemistry in the deep atmosphere and are fixed as our lower boundary condition. Chemical reactions and eddy mixing profiles are taken from Liang et al. (2003, 2004). Details of the model can be found in Liang et al. (2003, 2004 and references therein). For the simulation of the photochemistry of HD 209458b, we adopt the solar spectrum. For HD 189733b we use the spectrum of HD 22049, which is a K2 V star similar to HD 189733 (Segura et al. 2003). We have repeated our calculations for three temperature-pressure profiles (Fig. 1b), to test the sensitivity of our results to these assumptions. The modeled chemical abundances show a negligible dependence on temperature (see Liang et al. 2004).

The absorption coefficients in the MIR were estimated using a line-by-line model, LBLABC (Meadows \& Crisp 1996), that generates monochromatic gas absorption coefficients from molecular line lists-HITEMP (L. S. Rothman et al. 2007, in preparation)-for each of the gases present in the atmosphere.

\section{RESULTS}

Figure $1 a$ shows the molecular profiles of $\mathrm{H}_{2} \mathrm{O}, \mathrm{CO}, \mathrm{CH}_{4}$, $\mathrm{CO}_{2}$, and $\mathrm{C}_{2} \mathrm{H}_{2}$ for both planets HD 189733b and HD 209458b, calculated by the photochemistry model with the solar $\mathrm{C} / \mathrm{O}$ ratio as a boundary condition. On $\mathrm{HD} 189733 \mathrm{~b}, \mathrm{H}_{2} \mathrm{O}, \mathrm{CH}_{4}$, and $\mathrm{C}_{2} \mathrm{H}_{2}$ are more abundant in the upper atmosphere than $\mathrm{HD}$ 209458b, since HD 189733 is a later type star; therefore, the photodissociation processes occurring in the atmosphere of that planet are less significant.
Sensitivity to molecular abundances and $\mathrm{C} / \mathrm{O}$ ratio.-Figure 2 shows the predicted absorption signatures due to water vapor and $\mathrm{CO}$ on the planets HD 189733b and HD 209458b. The three plots compare the spectral absorptions of these two species when the $\mathrm{C} / \mathrm{O}$ ratio is solar (Fig. 2, standard case, solid line; see Fig. $1 a$ for mixing ratios) and when is below and above solar. As specific examples, we have assumed $\mathrm{H}_{2} \mathrm{O}$ to be 10 times more and at the same time CO 10 times less abundant than the standard case (dotted line), and vice-versa (dashed line). When we increase/diminish $\mathrm{H}_{2} \mathrm{O}$ and $\mathrm{CO}$ by a factor of 10 , the absorption is increased/decreased by a constant $\sim 0.03 \%$ through all of the the selected wavelength range. In these figures, we also show the signatures (open rhombi, squares, and triangles) relative to the three cases (standard, C/O ratio below and above solar) averaged over the Spitzer IRAC, IRS, and MIPS bandpasses (centered at 3.6, 4.5, $5.8,8,16$, and $24 \mu \mathrm{m}$; see Table 1 for the calculated absorptions). Water vapor has strong absorption lines through all of the selected spectral range in the MIR; the CO signature appears only in a narrower wavelength interval, where the IRAC $4.5 \mu \mathrm{m}$ bandpass is centered. When the $\mathrm{C} / \mathrm{O}$ ratio is above solar, the triangle is expected to appear above the rhombus in that band, indicating the strong $\mathrm{CO}$ contribution to the total absorption (Table 1, footnote "a"). Note that we included the species $\mathrm{CH}_{4}, \mathrm{CO}_{2}$, and $\mathrm{C}_{2} \mathrm{H}_{2}$ in our calculations, which strongly absorb in the MIR. However, their abundances are too small compared to $\mathrm{CO}$ and $\mathrm{H}_{2} \mathrm{O}$, and they are masked by these species. For example, we show in Figure $3 a$ the contribution due to $\mathrm{CO}_{2}$ in the case of $\mathrm{C} / \mathrm{O}$ above solar ratio. $\mathrm{CO}_{2}$ is increased by a factor of 10 here to follow $\mathrm{CO}$ behavior consistently with chemistry predictions (Liang et al. 2003). When $\mathrm{CO}_{2}$ is present, an increase in absorption of $\sim 0.02 \%$ is found in the band centered on $15 \mu \mathrm{m}$, and a very narrow peak reaching $0.07 \%$ is visible at shorter wavelengths. Although not negligible, the contribution due to $\mathrm{CO}_{2}$ is masked by $\mathrm{CO}$ and water.

Sensitivity to temperature.-The effects due to temperature variations (Fig. 3b) are negligible compared to the changes in molecular mixing ratios. Temperature plays a secondary role in the determination of the optical depth: it affects the absorption coefficients and the atmospheric scale heights (see eq. [2] in Ehrenreich et al. 2006). For HD 189733b, the discrepancy between the standard and the hot profile is less than $0.005 \%$, and between the standard and the very hot profile has a max- 

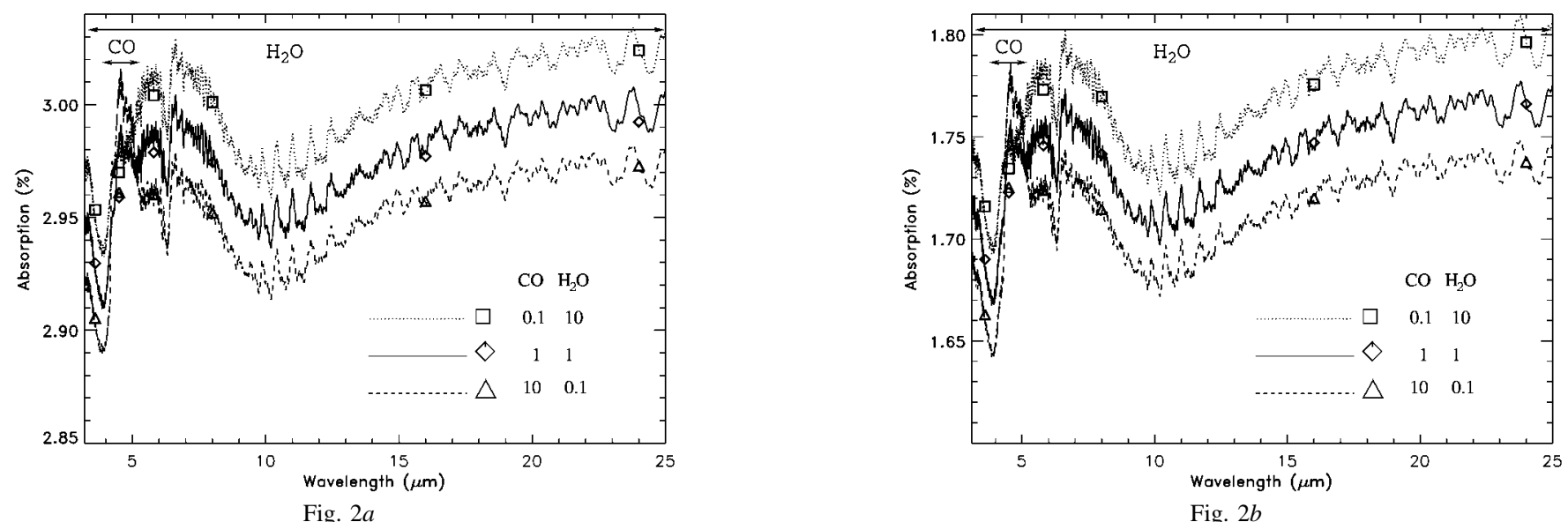

FIg. 2.-Modeled spectral absorptions of $\mathrm{H}_{2} \mathrm{O}$ and $\mathrm{CO}$ in the atmospheres of (a) HD 189733b and (b) HD 209458b during their transits. Solid line: Standard case, $\mathrm{C} / \mathrm{O}$ ratio $=$ solar (Fig. 1a). Dashed line: $\mathrm{CO}$ is overabundant and $\mathrm{H}_{2} \mathrm{O}$ underabundant by a factor of 10 . Dotted line: The opposite. Open triangles, squares, and rhombi indicate the spectral absorptions averaged over Spitzer IRAC, IRS, and MIPS bandpasses. Only in the $4.5 \mu \mathrm{m}$ band, where CO shows a strong absorption, do the triangle and rhombus overlap.

imum of $0.014 \%$ in the $15-30 \mu \mathrm{m}$ range (Fig. $3 b$ ). Analogous results are obtained for HD 209458b.

\section{DISCUSSION}

In our model we did not include the contribution of hazes or clouds (Ackerman \& Marley 2001; Lunine et al. 1989; Fortney et al. 2005). Due to their presence, the atmospheric optical depth might increase, partially masking the absorption features due to atmospheric molecules. In the case of water vapor and $\mathrm{CO}$, only clouds/hazes lying at altitudes higher than 1 bar might affect our results. Predictions of cloud/haze are particularly difficult for EGPs, since the few observations we have are not sufficient to constrain all the microphysics parameters. Moreover, the planetary limb observable during the star occultation might show the signatures of both the night and day side of those planets, which are presumably tidally locked (Iro et al. 2005). The thermal profiles, hence the condensate dynamics, might be very different on the two sides. Consequently, a more complete model able to predict cloud and haze location and optical characteristics, should contain a 3D dynamical simulation of the atmosphere. In this Letter we limit our simulations to the cloud-haze-free atmosphere, with the caveat that they might be perturbed by the possible presence (constant or variable) of optically thick particles in the atmosphere above 1 bar pressure.

The same considerations are valid for the thermal profiles. An extensive literature is available on T-P profiles for EGPs at pressures from $\sim 1$ bar to $10^{-4}$ to $10^{-6}$ bars, most recently including 3D dynamical effects (Showman \& Guillot 2002; Iro et al. 2005; Cho et al. 2006; Burrows et al. 2006). For transmission spectroscopy in the MIR, we also need to consider the contribution of the upper atmosphere. The T-P profiles calculated by Tian et al. (2005) and Yelle (2004) suggest that the trend for the atmospheric temperature is to increase in the exosphere. For our simulations, we use a T-P profile compatible with the lower atmosphere models cited above up to $10^{-3}$ to $10^{-4}$ bars, and then we consider three cases: the atmospheric temperature decreases up to $10^{-10}$ bars (standard profile), and the atmosphere is isothermal (hot, very hot profiles). Our results show that the differences among the spectra calculated with the three profiles are within $0.009 \%$ for $\lambda \leq 14 \mu \mathrm{m}$ and within $0.014 \%$ at longer wavelengths, so we are confident that our simulations will not significantly change using a more refined thermal structure.

Our model atmospheres extend to $10^{-10}$ bars, where nonlocal thermodynamic equilibrium (non-LTE) effects might occur (Kutepov et al. 1998). However, if we truncate our calculations to $10^{-5}$ bars, we obtain a maximum error of $\sim 0.02 \%$ at $30 \mu \mathrm{m}$ (no discrepancy for wavelengths shorter than $20 \mu \mathrm{m}$ ), indicating that our calculated absorptions in the LTE regime are correct at first-order approximation.

To detect the presence of $\mathrm{H}_{2} \mathrm{O}$ and $\mathrm{CO}$ on HD 189733b and HD 209458b in the MIR, an extra absorption of $\sim 0.15 \%$ is expected to be added to the $2.85 \%$ and $1.6 \%$ due to the optically thick disks at 1 bar atmospheric level. To estimate the chemical abundances, an accuracy of at least $0.03 \%$ is needed. By inspection of the relative absorption of the IRAC $4.5 \mu \mathrm{m}$ bandpass with respect to the others, we might be able to infer the $\mathrm{C} / \mathrm{O}$ ratio, but in this case an extremely high $\mathrm{S} / \mathrm{N}$ is required. HD 189733 is a bright $\mathrm{K} 0 \mathrm{~V}$ star of magnitude $K=5.5$. We estimate the brightness in the four IRAC bands to be of the order of $1850,1100,730$, and $400 \mathrm{mJy}$ at $3.6,4.5,5.8$, and $8 \mu \mathrm{m}$, respectively. For HD 209458, a G0 V star with $K$-magnitude of 6.3 , the IRAC predicted fluxes are 878, 556, 351, and $189 \mathrm{mJy}$. According to these numbers, a better S/N should be obtainable for HD 189733b (Deming et al. 2006), and this makes HD 189733b a better candidate for observations.

TABLE 1

Calculated Absorptions Averaged over IRAC, IRS, AND MIPS BANDPASSES

\begin{tabular}{|c|c|c|c|c|c|c|c|}
\hline HD & $\begin{array}{l}\mathrm{C} / \mathrm{O} \\
\text { Ratio }\end{array}$ & $3.6 \mu \mathrm{m}$ & $4.5 \mu \mathrm{m}$ & $5.8 \mu \mathrm{m}$ & $8 \mu \mathrm{m}$ & $16 \mu \mathrm{m}$ & 24 \\
\hline 189733b & $<$ Solar & 2.953 & 25 & 30 & 30 & 3.0 & 3.0 \\
\hline 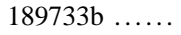 & Solar & 2 & & & 4 & & 2.9 \\
\hline b & & & & & & & 2.97 \\
\hline & $<$ & & & & & & 76 \\
\hline $209458 b$ & Sol & 1.69 & 1.7 & O & 1.741 & 1.747 & 1.7 \\
\hline $209458 b$ & $>$ Solar & 1.663 & $1.725^{\mathrm{a}}$ & 1.724 & 1.714 & 1.720 & 1.738 \\
\hline
\end{tabular}

Note. - We recall that $2.85 \%$ and $1.6 \%$ are the nominal absorptions due to the optically thick disks at 1 bar atmospheric level.

${ }^{a} \mathrm{C} / \mathrm{O}$ ratio is above solar and $\mathrm{CO}$ strongly contributes in the $4.5 \mu \mathrm{m}$ IRAC band. 

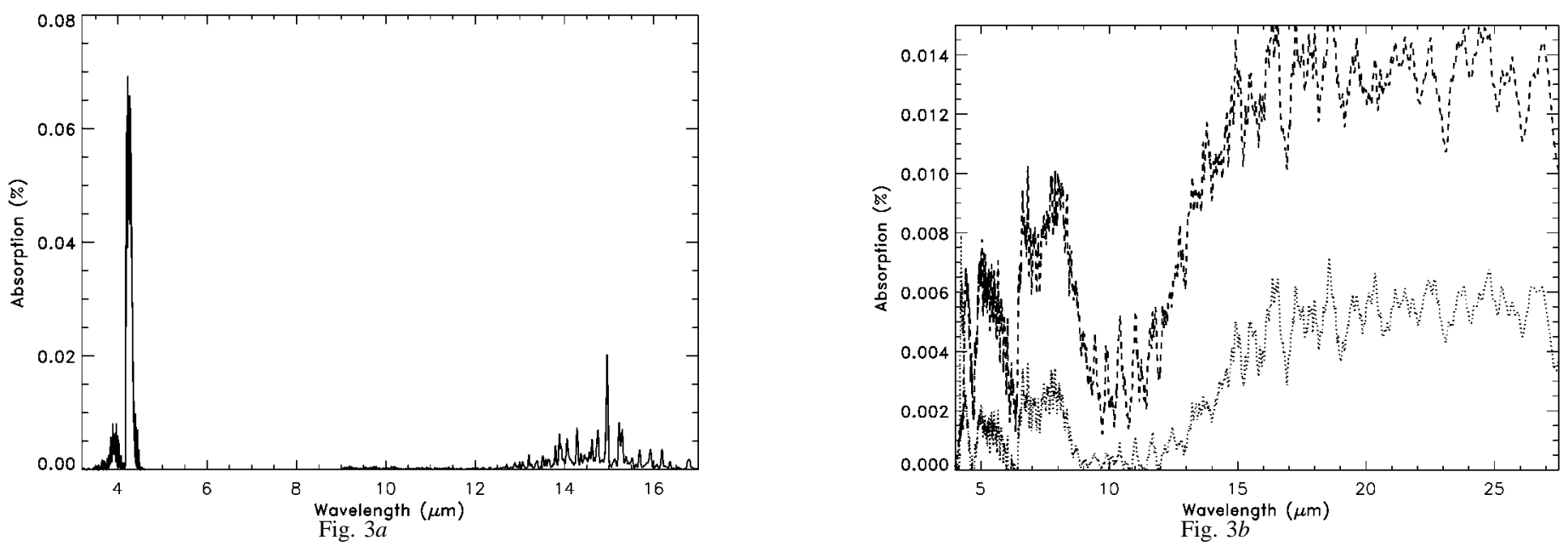

FIG. 3.- (a) Residue spectrum from difference of two curves. Dashed line plot in Fig. 2 (top) with and without the contribution of $\mathrm{CO}_{2}$. $\mathrm{CO}_{2}$ is assumed to be, like CO, overabundant by a factor of 10. (b) Residue spectra from difference of two curves. Dotted line: Difference from hot and standard profiles. Dashed line: Difference from very hot and standard profiles (Fig. 1b).

\section{CONCLUSIONS}

We have presented here simulations of transmission spectra of two extrasolar giant planets during their transit in front of their parent star. We estimate an excess absorption in the IR of up to $0.15 \%$ for HD 189733 b and up to $0.12 \%$ for HD 209458 b (C/O ratio solar), in addition to the nominal $2.85 \%$ and $1.6 \%$ absorptions measured at shorter wavelengths. If water were far less abundant, other species might be observable, depending on their mixing ratios. Among them, $\mathrm{CO}_{2}, \mathrm{CH}_{4}$, and $\mathrm{C}_{2} \mathrm{H}_{2}$ are the best candidates.

According to our simulations, transmission spectra of EGPs in the MIR are sensitive to molecular abundances and less to temperature variations. Temperature influences the transmission spectrum by way of its influence on the atmospheric scale height, as discussed by Brown (2001), and on the absorption coefficients. If water vapor and $\mathrm{CO}$ are as abundant as photo- chemical models predict, we expect that they can be detected with the Spitzer IRAC, IRS, and MIPS instruments and with future telescopes like JWST. Moreover, if an accuracy of $0.03 \%$ is obtainable, future observations may give a first direct estimate of $\mathrm{H}_{2} \mathrm{O}$ and $\mathrm{CO}$ abundances in the upper atmosphere of EGPs and possibly-depending on their mixing ratios-a constraint on $\mathrm{CO}_{2}, \mathrm{CH}_{4}$, and $\mathrm{C}_{2} \mathrm{H}_{2}$.

We would like to thank the anonymous referee for his help to improve the Letter, L. S. Rothman for having provided the HITEMP data list, and R. Ferlet, J. M. Désert, F. Bouchy, G. Hébrard, C. D. Parkinson, A. Noriega-Crespo, and S. Carey, for their valuable inputs. G. T. is currently supported by the European Space Agency, and M.-C. L. and Y. L. Y. by NASA grant NASA5-13296 to Caltech.

\section{REFERENCES}

Ackerman, A. S., \& Marley, M. S. 2001, ApJ, 556, 872

Bouchy, F., et al. 2005, A\&A, 444, L15

Brown, T. M. 2001, ApJ, 553, 1006

Burrows, A., Sudarsky, D., \& Hubbard, W. B. 2003, ApJ, 594, 545

Burrows, A., Sudarsky, D., \& Hubeny, I. 2006, ApJ, 650, 1140

Butler, R. P., et al. 2006, ApJ, 646, 505

Charbonneau, D., Brown, T. M., Latham, D. W., \& Mayor, M. 2000, ApJ, 529, L45

Charbonneau, D., Brown, T. M., Noyes, R. W., \& Gilliland, R. L. 2002, ApJ, 568, 377

Cho, J. Y. K., Menou, K., Hansen, B. M. S., \& Seager, S. 2006, ApJ, submitted (astro-ph/0607338)

Deming, D., Brown, T. M., Charbonneau, D., Harrington, J., \& Richardson, L. J. 2005a, ApJ, 622, 1149

Deming, D., Harrington, J., Seager, S., \& Richardson, L. J. 2006, ApJ, 644, 560

Deming, D., Seager, S., Richardson, L. J., \& Harrington, J. 2005b, Nature, 434, 740

Ehrenreich, D., Tinetti, G., Lecavelier des Etangs, A., Vidal-Madjar, A., \& Selsis, F. 2006, A\&A, 448, 379

Fortney, J. J., Marley, M. S., Lodders, K., Saumon, D., Freedman, R. 2005, ApJ, 627, L69

Fortney, J. J., Sudarsky, D., Hubeny I., Cooper, C. S., Hubbard, W. B., Burrows, A., \& Lunine, J. I. 2003, ApJ, 589, 615

Gardner, J. P., et al. 2006, Space Sci. Rev., 123, 485

Henry, G. W., Marcy, G. W., Butler, R. P., \& Vogt, S. S. 2000, ApJ, 529, L41

Iro, N., Bézard, B., \& Guillot, T. 2005, A\&A, 436, 719

Kuchner, M., \& Seager, S. 2006, ApJ, submitted (astro-ph/0504214)
Kutepov, A. A., Gusev, O. A., \& Ogibalov, V. P. 1998, J. Quant. Spectrosc. Radiat. Transfer, 60, 199

Lecavelier des Etangs, A., Vidal-Madjar, A., McConnell, J. C., \& Hébrard, G. 2004, A\&A, 418, L1

Liang, M.-C., Parkinson, C. D., Lee, A. Y.-T., Yung, Y. L., \& Seager, S. 2003, ApJ, 596, L247

Liang, M.-C., Seager, S., Parkinson, C. D., Lee, A. Y.-T., \& Yung, Y. L. 2004, ApJ, 605, L61

Lunine, J. I., Hubbard, W. B., Burrows, A., Wang, Y.-P., \& Garlow, K. 1989, ApJ, 338, 314

Mazeh, T., et al. 2000, ApJ, 532, L55

Meadows, V. S., \& Crisp, D. 1996, J. Geophys. Res., 101, 4595

Richardson, L. J., Deming, D., \& Seager, S. 2003a, ApJ, 597, 581

Richardson, L. J., Deming, D., Wiedemann, G., Goukenleuque, C., Steyert, D., Harrington, J., \& Esposito, L. W. 2003b, ApJ, 584, 1053

Richardson, L. J., Harrington, J., Seager, S., \& Deming, D. 2006, ApJ, 649, 1043

Schneider, J. 2006, The Extrasolar Planets Encyclopedia, http://exoplanet.eu

Seager, S., Richardson, L. J., Hansen, B. M. S., Menou, K., Cho, J. Y.-K., \& Deming, D. 2005, ApJ, 632, 1122

Seager, S., \& Sasselov, D. D. 2000, ApJ, 537, 916

Segura, A., Krelove, K., Kasting, J. F., Sommerlatt, D., Meadows, V., Crisp, D., Cohen, M., \& Mlawer, E. 2003, Astrobiology, 3(4), 689

Showman, A. P., \& Guillot, T. 2002, A\&A, 385, 166

Tian, F., Toon, O. B., Pavlov, A. A., \& De Sterck, H. 2005, ApJ, 621, 1049

Vidal-Madjar, A., Lecavelier des Etangs, A., Désert, J. M., Ballester, G. E., Ferlet, R., Hébrard, G., \& Mayor, M. 2003, Nature, 422, 143

Vidal-Madjar, A., et al. 2004, ApJ, 604, L69

Yelle, R. 2004, Icarus, 170, 167 